\title{
A Randomised Placebo-Controlled Trial of Topical Cysteamine Therapy in Patients with Nephropathic Cystinosis
}

\author{
JOHN A. BRADBURY ${ }^{1}$, JEAN-PIERRE DANJOUX, JEAN VOLLER, MICHAEL \\ SPENCER $^{2}$, TREVOR BROCKLEBANK ${ }^{3}$
}

Leeds

\begin{abstract}
Summary
Five patients with nephropathic cystinosis were evaluated to assess the ability of topical cysteamine to clear corneal cystine crystals. All patients were randomised to receive topical cysteamine $0.2 \%$ six times a day in one eye with normal saline in the other eye as a control.

All five patients showed some improvement in visual symptoms (photophobia, blepharospasm and visual acuity) together with an improvement in corneal crystal density. Three of these also had an improvement in Snellen visual acuity and contrast sensitivity.
\end{abstract}

Cystinosis is an autosomal recessive metabolic disorder in which non-protein cystine crystals accumulate within cellular lysosomes due to a defect in lysosomal cystine transport (1).

The systemic clinical manifestations include severe growth retardation, renal failure, hepatosplenomegaly and hypothyroidism.

Ocular involvement includes deposition of crystals in corneae and conjunctivae (Fig. 1) which worsens with age. This may lead to photophobia, blepharospasm, corneal erosions and reduced visual acuity. There may also be iris thickening and impairment of rod and cone function $(1,2)$.

Oral cysteamine has been shown to deplete the intracellular cystine crystals producing a clinical improvement in growth and renal function but not in ocular function (2). Topical ocular cysteamine in the range of $0.1 \%$ to
$0.2 \%$ has been shown to reduce the amounts of intracorneal cystine crystals in some patients and improve visual symptoms $(1,3,4,5)$.

The purpose of this study was to assess the ability of cysteamine eye drops to deplete the corneae of cystine crystals and document any improvement in visual function in children with proven cystinosis.

\section{Patients and methods}

Six patients with known cystinosis who attended the Paediatric Nephrology Clinic at St James's Hospital, Leeds were entered into the study. One 14 year old patient withdrew two weeks after treatment was started, requesting no further participation. The study period was from December 1989 to May 1990.

All patients were diagnosed as having cystinosis based on a typical clinical presentation and confirmed by either fibroblast culture or

From the Department of Ophthalmology (1), Pharmacy (2) and Paediatrics (3), St James University Hospital, Leeds.

Correspondence to: Mr. J. Bradbury, Department of Ophthalmology, The General Infirmary at Leeds, Great George Street, Leeds LS1 3EX, West Yorkshire. 


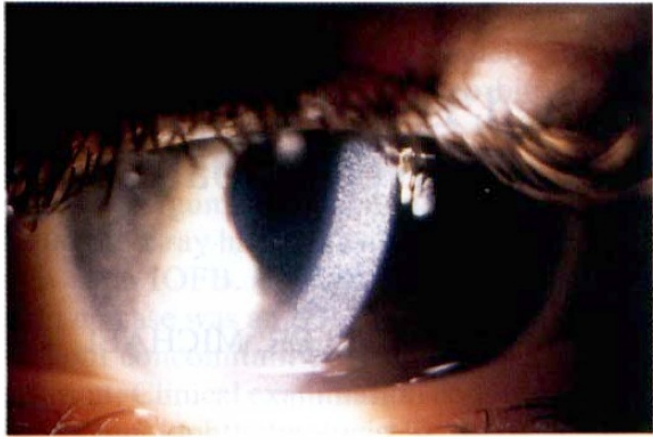

Fig. 1. Slit-lamp photography of the left eye of an 8-year-old boy $(A W)$ with cystinosis. There are multiple crystals of non-protein cystine deposited throughout the corneal stroma.

the demonstration of a leucocyte cystine concentration greater than $3 \mathrm{n}$. mol. half cystine per milligram of protein (normal concentration less than $0.2 \mathrm{n}$. mol. half cystine per milligram of protein).

All patients had corneal cystine crystals when entered into the study and all but one (AS) was receiving oral cysteamine.

A double masked, randomised, placebocontrolled trial was started using two sets of coded eye drops six times a day in each eye. One set contained $0.2 \%$ cysteamine hydrochloride in $0.9 \%$ sodium chloride with disodium edetate $0.02 \%$ as a stabiliser. The other set contained sodium chloride $0.9 \%$. The eye drops were prepared by the Pharmacy Department at St James's Hospital, Leeds, who also administered the randomisation codes.

At each monthly visit, the following parameters were measured:

1 Visual Acuity

By illuminated Snellen's Chart at six metres wearing full distance correction.

2 Contrast Sensitivity

Using the Vistech contrast sensitivity chart at 10 feet under recommended conditions. (Vistech Consultants Inc)

\section{Slit-Lamp Biomicroscopy}

To assess the number of corneal crystals which was scored on an arbitrary scale of zero to four plus of crystals on 0.5 increments and noting any other anterior segment pathology.

Although the crystal density scores were arbi- trary and subjective, two scores were made by independent observers (JAB, JPD) who were both masked. The final score was then an average from both observers.

4 Subjective Assessment of photophobia, pain, blepharospasm and visual acuity for each eye.

Each patient kept a record of the number of drops instilled in each eye every day which was declared at each hospital visit.

A slit-lamp photograph using a Zeiss photo slit-lamp (Carl Zeiss \& Co, Oberkochen, West Germany) was taken of each eye at every visit.

The patients were assessed monthly for a period of six months at which time the code was broken.

Fully informed consent was obtained from both the patient and parent(s) in each case before the start of the study.

\section{Results (Table 1)}

All patients showed a subjective improvement in visual symptoms such as photophobia, pain, blepharospasm and visual acuity in the eye receiving topical cysteamine.

On closer questioning, there was also a small improvement in visual symptoms in the eye receiving topical sodium chloride $0.9 \%$ in four out of five cases. One case (AW) noted visual improvement only in the eye receiving topical cysteamine.

There were no substantial increases in visual acuity for any patient in the study although patients 1, 2 and 3 (And.W, Ang.W, JS) showed a slight improvement in visual acuity in the treated compared with the untreated eye which mirrored the improvements noted with other parameters.

Contrast sensitivity (Fig. 2) seemed to mirror the improvements seen with crystal density score, visual symptoms, and visual acuity seen in patients 1,2 and 3 . This was not seen in patient 4 , and patient 5 could not cooperate with the test.

All patients showed some improvement in crystal density which preceded improvements in all parameters in patients 1,2 and 3 and a subjective improvement in visual symptoms in patients 4 and 5 .

Patient 5 was our eldest and most severely affected subject with the highest cystine crystal density scores throughout the study. 
Table I Summary of results of 5 patients receiving cysteamine $0.2 \%$ drops in one eye and sodium chloride $0.9 \%$ in the other eye. All show some improvement in the eye receiving cysteamine drops.

\begin{tabular}{|c|c|c|c|c|c|c|c|c|}
\hline \multirow{3}{*}{$\begin{array}{l}\text { Case } 1 \\
\text { AW }\end{array}$} & \multicolumn{3}{|c|}{$\begin{array}{l}\text { Visual Acuity } \\
0-6 \text { months }\end{array}$} & \multirow{3}{*}{$\begin{array}{c}\text { Contrast Sensitivity } \\
\text { Improved Right Eye }\end{array}$} & \multicolumn{2}{|c|}{$\begin{array}{c}\text { Crystal Density Score } \\
\text { 0-6 months }\end{array}$} & \multirow{3}{*}{$\begin{array}{c}\begin{array}{c}\text { Subjective } \\
\text { Symptoms }\end{array} \\
\uparrow \text { VA right eye }\end{array}$} & \multirow{3}{*}{$\begin{array}{c}\begin{array}{c}\text { Eye Receiving } \\
\text { Cysteamine }\end{array} \\
\text { Right }\end{array}$} \\
\hline & $\mathrm{R}$ & $6 / 5$ & $6 / 6$ & & +3 & +2 & & \\
\hline & $\mathrm{L}$ & $6 / 5$ & $6 / 9$ & & +3 & +3 & & \\
\hline Case 2 & $\mathrm{R}$ & $6 / 12$ & $6 / 9$ & Improved Right Eye & +3 & +2.5 & Improved BE & Right \\
\hline Ang.W & $\mathrm{L}$ & $6 / 6$ & $6 / 6$ & & +3 & +3 & $\mathrm{R}>\mathrm{L}$ & \\
\hline Case 3 & $\mathrm{R}$ & $6 / 4$ & $6 / 6$ & Improved Left Eye & +3 & +3 & $\uparrow$ VA photophobic & Left \\
\hline JS & $\mathrm{L}$ & $6 / 6$ & $6 / 5$ & & +3 & +1.5 & + less blepharospasm & \\
\hline Case 4 & $\mathrm{R}$ & $6 / 6$ & $6 / 6$ & No Change & +3 & +2.5 & Less photophobic & Right \\
\hline AS & $\mathrm{L}$ & $6 / 6$ & $6 / 6$ & & +3 & +3.0 & $+\uparrow \mathrm{VA} \mathrm{R}>\mathrm{L}$ & \\
\hline Case 5 & $\mathrm{R}$ & $6 / 36$ & $6 / 36$ & No Measure Possible & +4 & +3.5 & Slightly less photophobic & Right \\
\hline RS & $\mathrm{L}$ & $6 / 36$ & $6 / 36$ & & +4 & +4.0 & + better vision $\mathrm{R}>\mathrm{L}$ & \\
\hline
\end{tabular}

\section{Case Histories}

Case $1(A W)$. Male, age 8 years

Cystinosis was diagnosed at age one year, an elder sister also had cystinosis (and is included in this study). He commenced Chronic Ambulatory Peritoneal Dialysis (CAPD) in 1988 for chronic renal failure and had a successful renal transplant in 1989 . He had been taking oral cysteamine since 1987.

On enrolment into the study, there were $3+$ of crystals in each cornea. After the study of six months, the right eye showed $2+$ of crystals compared with $3+$ for the left eye. The code was broken to reveal the right eye had received the cysteamine $0.2 \%$ drops.

At the beginning of the study, the patient had little blepharospasm or pain which was unchanged at the end of the study, although there was a subjective improvement in visual acuity in the right eye.

Compliance throughout the study was good, with drops being taken five or six times daily most days although only outside school hours. This was due to the school teacher's refusal to administer any medication.

\section{Case 2 (Ang.W). Female, age 13 years}

The elder sister of Case 1 was diagnosed as having cystinosis at age two years. CAPD was started at age nine years for chronic renal failure. Two years later, she had a renal transplant. Unfortunately, chronic rejection meant that CAPD was restarted in 1989. She had been on oral cysteamine since 1987 .

Over the study period there was some improvement in crystal density, relative visual acuity and contrast sensitivity in the eye receiving topical cysteamine. There was a general improvement in visual symptoms especially in the left eye.

Compliance throughout the study period was patchy due to the long-standing illness. Drops were only taken as her protocol (six times daily) for four months of the study period. For a three week period, no drops were taken at all. As in Case 1, no drops were administered while at school.

Case 3 (JS). Male, age 9 years

Cystinosis was diagnosed at age two years. Renal function is only mildly impaired and does not require dialysis. Oral cysteamine was started in 1987 and is well tolerated.

During the study period there was a subjective improvement in blepharospasm, photophobia and visual acuity. Objectively there was an improvement in Snellen visual acuity, contrast sensitivity and crystal density in the left eye. The left eye had been receiving topical cysteamine.

Compliance was not constant. He was fully compliant for only four out of the six month study period.

Case $4(A S)$. Male, age 10 years

Cystinosis was diagnosed at age two years. CAPD was started in 1988 for chronic renal failure and a successful renal transplant performed in 1989. Oral cysteamine was stopped after a short period of administration in 1987 because of an urticarial reaction.

A subjective improvement in vision and photophobia was seen in the right eye together with a decrease in corneal crystal density. There was no improvement in visual 


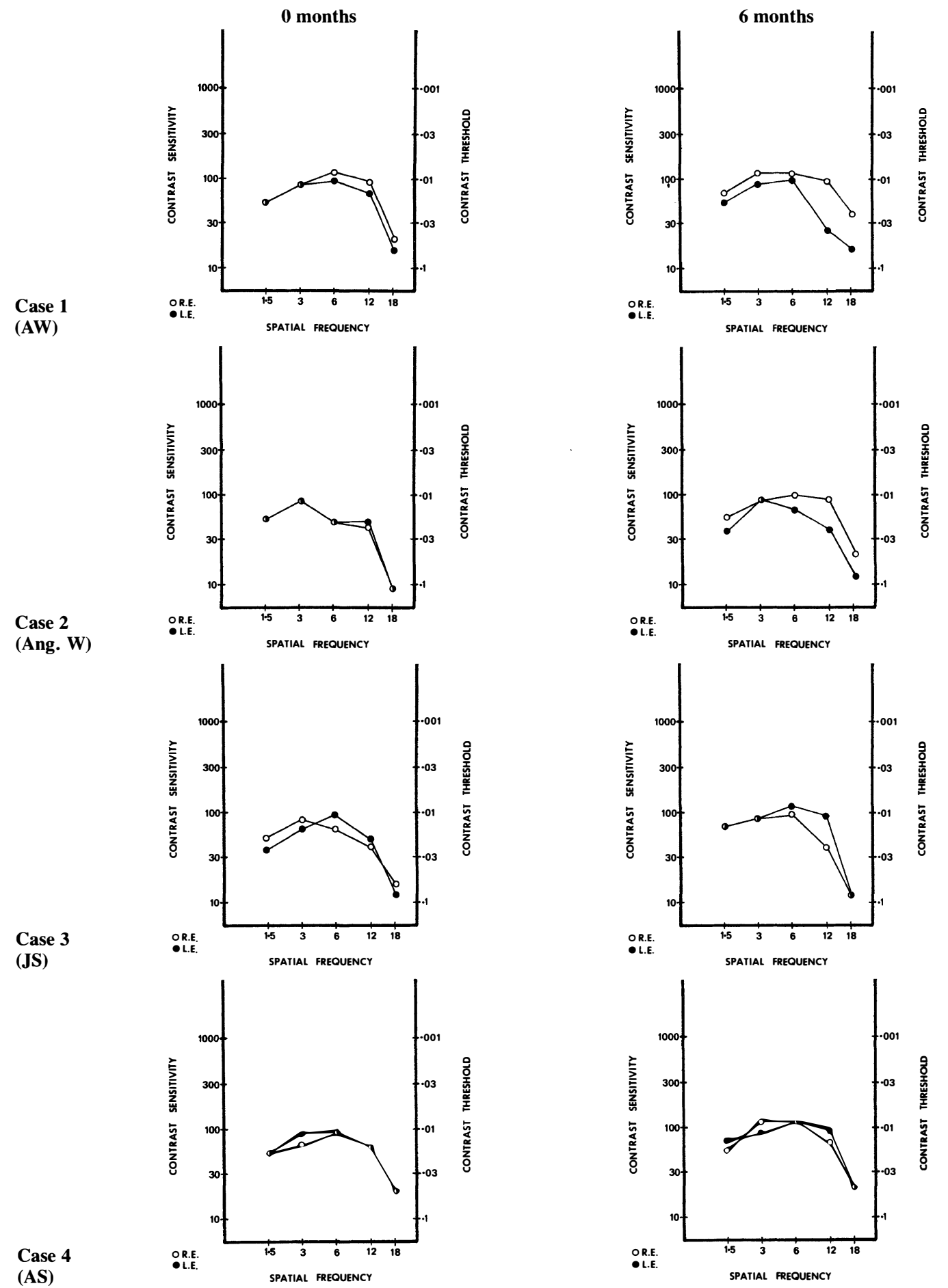

(AS)

Fig. 2. Contrast sensitivity of 4 subjects with cystinosis measured by Vistech contrast sensitivity charts at 10 feet (Vistech Consultants Inc). Patients 1, 2 and 3 showed improvement in contrast sensitivty during the study period in the eye receiving topical cysteamine $0.2 \%$. Patient 4 showed no improvement. The contrast sensitivity charts on the left were taken at the beginning of the study, the charts on the right at the completion of the study. 
acuity or contrast sensitivity. When the code was broken, the right eye had been receiving topical cysteamine.

Compliance was very good throughout the trial period. Drops were administered by a school teacher during school time.

\section{Case 5 (RS). Female, age 16 years}

Cystinosis was diagnosed at age 12 months. CAPD was commenced in 1982 for chronic renal failure. An initially successful renal transplant was rejected and CAPD restarted in 1987. She received a further renal transplant in 1990 during the trial period. She had been taking oral cysteamine since 1986 .

Our oldest and most severely affected patient (RS) showed only slight improvement in subjective symptoms such as photophobia and visual acuity, together with some decrease in crystal density in the right eye-the eye receiving topical cysteamine. No improvement in Snellen visual acuity was seen.

Compliance was very good considering that her general poor health and intense photophobia made drop administration very difficult. The drops were administered four times daily throughout the study.

\section{Discussion}

There was a subjective improvement in ocular symptoms (photophobia, pain, blepharospasm and visual acuity) in all our subjects over the study period and this was reflected in decreased corneal crystal density scores. However, further objective evidence of improvement was available for only three cases. In all cases subjective improvement occurred before objective improvements in visual function. Improvement in ocular symptomatology have been noted by other authors as the first change seen in topical cysteamine therapy (5). A possible explanation is that the ocular discomfort is due to severe anterior stromal disease stimulating the sensory nerve endings at the level of the basal epithelial cells. Topical cysteamine therapy reduces the anterior stromal crystals first, resulting in pain relief and probably improvement in blepharospasm and photophobia.

The visual acuity measurements varied from visit to visit and depended upon a number of factors including the general health of the patient and the strength of the sunlight. However, if the visual acuity in the untreated eye is taken as the benchmark for each visit, then cases 1, 2 and 3 showed a small improvement in visual acuity. The visual acuity improvement may be largely due to the improvement in photophobia and blepharospasm rather than an improvement in corneal transparency. Both objective and subjective improvements in visual acuity have been shown by other authors $(3,4,5)$.

Contrast sensitivity has been shown to be impaired in patients with cystinosis. Katz (5) showed that contrast sensitivity is reduced across all frequencies but especially at higher frequencies and is largely secondary to the corneal crystals and associated oedema. This reduced contrast sensitivity is also associated with increased glare (6). Improvement in contrast sensitivity mirrored the subjective and objective improvement in vision seen in three out of the four patients measured. The other patient measured showed only a subjective improvement in vision and visual symptoms together with a decrease in the crystal density in the eye receiving topical cysteamine therapy. The improvement seen in contrast sensitivity, although small, is encouraging as this indicates a true improvement in visual function rather than observing fluctuations in a disease process.

Kaiser-Kupfer (4) showed improvement in visual acuity and crystal clearance in eight out of 16 patients receiving topical cysteamine aged under 42 months and only showed similar improvement in two out of nine patients receiving topical cysteamine in a group aged four to 23 years. All were receiving cysteamine $0.5 \%$ hourly for at least four months. In contrast, we showed some improvement in three patients and minimal improvement in two patients, aged between nine and 16 years, receiving $0.2 \%$ cysteamine six times a day (or less) for six months. Compared to the group that did best in the KaiserKupfer study, our patients were older and received a weaker concentration of cysteamine less frequently, so were less likely to have a rapid corneal crystal clearance. Unfortunately, Kaiser-Kupfer's study using cysteamine at the $0.5 \%$ concentration was published after the start of our study. Compliance was a 
problem for all of our subjects, both because of photophobia and blepharospasm (which improved with administration of the cysteamine drops) and the unwillingness (in certain cases) of teachers to administer the drops whilst the subject was at school.

In conclusion, we found a small improvement in visual function in three patients and minimal improvement in two patients receiving topical cysteamine $0.2 \%$ for six months. All patients in the study wanted to continue with the treatments in both eyes as they believed there was significant improvement in visual function.

We suggest administering cysteamine eye drops at the $0.5 \%$ concentration at least six times a day to obtain the quickest improvement in visual function. Any significant improvement in visual function will occur only after four to six months of compliant treatment. The older the patient, the longer the required treatment.

There is a reasonable but unproven case for prophylactic treatment of those cases shown to have cystinosis but who have not yet developed corneal crystals.

The development of an ointment or sustained release preparation of cysteamine would help compliance.
A large prospective study (which may need to be multicentre) using the cysteamine $0.5 \%$ drops for a longer period may yield clearer results.

\section{References}

${ }^{1}$ Gahl WA, Thoen JA, Schneider JA, et al.: Cystinosis: Progress in Prototypic Disease. Ann Int Med 1988; 109: 557-69.

${ }^{2}$ Gahl WA, Reed GF, Thoene JG, et al.: Cysteamine Therapy for Children with Nephropathic Cystinosis. New Eng J Med 1987; 316: 971-7.

${ }^{3}$ Kaiser-Kupfer MI, Fujikawa L, Kuwabara T: Removal of Corneal Crystals by Topical Cysteamine in Nephropathic Cystinosis. New Eng J Med 1987; 316: $775-9$.

${ }^{4}$ MacDonald ZM, Noel LP, Mintsioulis G, et al.: The Effect of Topical Cysteamine Drops on Reducing Crystal Formation within the Cornea of Patients Affected by Nephropathic Cystinosis. JPOS 1990; 27: 272-4.

${ }^{5}$ Kaiser-Kupfer MI, Gazzo MA, Datiles MB, et al.: A Randomized Placebo Controlled Trial of Cysteamine Eye Drops in Nephropathic Cystinosis. Arch Ophthalmol 1990; 108: 689-3.

${ }^{6}$ Katz B, Melles RB, Schneider JA: Contrast Sensitivity Function in Nephropathic Cystinosis. Arch Ophthalmol 1987; 105: 1667-9.

${ }^{7}$ Katz B, Melles RB, Schneider JA. Glare Disability in Nephropathic Cystinosis. Arch Ophthalmol 1987; 105: $1970-1$. 